\title{
Predator-Prey Relationships in a Model for the Activated Process
}

\author{
RAYMOND P. CANALE, The University of Michigan, Department \\ of Civil Engineering, Ann Arbor, Michigan 48104
}

\section{Summary}

The primary objective of this paper was to develop a mathematical description for the food chain,

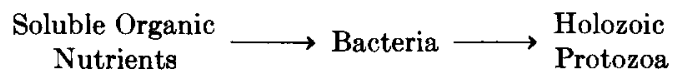

Because of the interdependence of the elements in this food chain, continuous oscillations among the variables are possible. A set of three differential equations was obtained to describe the above system in a continuously fed stirred tank reactor. The differential equations obtained were examined to characterize the possible types of solutions. A limit cycle solution was obtained for some values of the system parameters.

\section{INTRODUCTION}

The biological community of an efficient wastewater treatment plant employing the activated sludge process is extremely diverse. Microscopic examination of sludge often reveals bacteria, fungi, protozoa, and rotifers. Less numerous organisms which occur are crustaceans and nematodes.

Bacteria are the primary agents responsible for the stabilization of dissolved organic material in the activated sludge process. However, chemosynthetic Mastigophora and fungi obtain food in a manner similar to that of the bacteria, that is, by transport of soluble nutrients across the cell membrane. Thus, these organisms compete with bacteria for their food. Lackey ${ }^{1}$ studied the distribution of chemosynthetic Mastigophora in activated sludge as well as in other biological treatment units. The free-swimming and stalked Ciliata, 
as well as the rotifers, rely mainly on bacteria as a source of food. The Ciliata, their occurrence and importance in activated sludge, have been the subjects of research by many workers. It was established by Jenkins ${ }^{2}$ and Lackey ${ }^{1}$ and later by Baines et al. ${ }^{3}$ that there is little correlation between protozoan populations and the degree of purification in activated sludge treatment plants. In a more recent work, McKinney and Gram ${ }^{4}$ showed in laboratory studies that many free-swimming Ciliata are capable of clearing a cloudy suspension of bacteria. Other important contributions concerning the role of protozoa in activated sludge include: Cramer, ${ }^{5}$ Reynoldson, ${ }^{6}$ and Heukelikian and Gurbaxani. ${ }^{7}$

The microorganism population of activated sludge is dynamic in nature. Competition for soluble food occurs among the bacteria, the fungi, and the chemosynthetic Mastigophora. Holozoic freeswimming and stalked Ciliata and the rotifers are competing while preying on the saprobic organisms. An excellent qualitative discussion of these ideas was presented by McKinney and Gram. ${ }^{4}$ The present study is concerned with the development of a mathematical model to aid in obtaining quantitative information regarding some aspects of predator-prey relationships in continuously fed biological reactors.

\section{THE MATHEMATICS OF CONTINUOUS CULTURE OF MICROORGANISMS}

It is generally agreed that various species of bacteria are the most important microorganisms responsible for the removal of organic material in biological waste treatment processes. Thus, as a first approximation, many workers have neglected the effects of all organisms other than bacteria. The theory associated with this model was published by Monod (1942) and later by Herbert et al. ${ }^{8}$ In this model, a sterile growth medium is fed continuously into a completely mixed culture vessel at a constant volumetric flow rate $Q$. The culture vessel has a constant volume $V$. The culture vessel contains bacterium with a concentration, $b$ (dry weight of bacterium per unit volume). The rate of increase of bacterial concentration, $r_{b}$, is only a function of the limiting nutrient concentration in the growth medium $s$ and the concentration of bacterium $b$. The units of $s$ are weight of limiting nutrient per unit volume. Several investigators such as Monod (1942), Downing and Wheatland, ${ }^{9}$ Steward and 
Ludwig, ${ }^{10}$ and Rich ${ }^{11}$ have proposed the use of eq. (1) for relating $r_{b}$ and $s$.

$$
r_{b}=M \frac{s}{K+s} b
$$

In eq. (1) $M$ is the specific growth rate and has units (time ${ }^{-1}$ ), and $K$ is a saturation constant which is equal to the limiting nutrient concentration when $r_{b} / b=M / 2$.

Associated with the bacterial concentration increase is a decrease in the limiting nutrient concentration. Monod (1942) presented a simple relationship between the growth $r_{b}$, and the substrate utilization rate $r_{s}$, as shown by eq. (2):

$$
r_{b}=-Y r_{s}
$$

where $Y$ is the yield constant defined by eq. (3).

$$
Y=\frac{\text { weight of bacteria formed }}{\text { weight of substrate used }}
$$

A mass balance for the limiting substrate concentration in the culture vessel with an influent concentration of $s_{I}$ gives eq. (4) using eqs. (1), (2), and (3).

Rate of increase

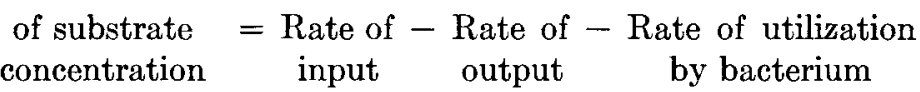

$$
\frac{d s}{d t} \quad=\frac{Q}{V} s_{I}-\frac{Q}{V} s-\frac{M}{Y} \frac{s}{K+s} b
$$

A similar equation for the change in concentration of the bacterium is eq. (5).

$$
\begin{aligned}
& \text { Rate of increase of Rate of Rate of } \\
& \text { bacterium concentration }=\text { growth }- \text { output } \\
& \frac{d b}{d t} \quad=M \frac{s}{K+s} b-\frac{Q}{V} b
\end{aligned}
$$

At steady state, $d s / d t=d b / d t=0$. The substrate concentration at steady state $s_{0}$ is obtained from eq. (5).

$$
s_{0}=\frac{K}{(M V / Q)-1}
$$


The bacterial concentration at steady state $b_{0}$ is obtained from eq. (4).

$$
b_{0}=Y\left(s_{I}-s_{0}\right)=Y\left[s_{I}-\frac{K}{(M V / Q)-1}\right]
$$

As shown by Koga and Humphrey, ${ }^{12}$ these values of $s_{0}$ and $b_{0}$ represent stable equilibrium points of eqs. (4) and (5) provided $Q / V$ is less than $M\left[s_{I} /\left(K+s_{I}\right)\right]$. Thus, the simple mathematical model proposed by Monod predicts that the continuously fed culture vessel will have steady state bacterium and nutrient concentrations given by $s_{0}$ and $b_{0}$.

Equations (6) and (7) represent a simple model for bacterial concentration and substrate concentration in a completely mixed reactor. Martin and Washington ${ }^{13}$ showed that the effects of cell lysis and death are not important in the above mathematical model. Recently, Washington ${ }^{14}$ showed that $Y$, rather than being a constant fraction, is actually a function of the dilution rate $Q / V$. Reynolds and Yang ${ }^{15}$ used equations similar to eqs. (4) and (5) and included the effects of sludge recirculation and endogenous respiration. However, the solution of such equations with these additional complications is also constant steady state values for $s$ and $b$.

Cassell et al. ${ }^{16}$ investigated the use of Monod's theory for activated sludge processes. The following summarizes the results of their findings:

This study was concerned mainly with the dynamics of mixed cultures as a function of flow rate. Laboratory experiments were performed with natural, mixed cultures propagated continuously under controlled aerobic conditions. Effects of flocculation and sedimentation, although essential to waste treatment plants, were eliminated purposely by operating the laboratory units without settling and sludge return. Under these circumstances, it was possible to test the usual implicit assumption made in theoretical anzlysis of completely mixed systems, namely that mixed microbial cultures behave essentially as continuous pure cultures reaching a stable equilibrium, or the so-called steady state. The experiments revealed that mixed culture systems are very dynamic and that phenomena of selection and predomination strongly influence their behavior contrary to the assumption of steady state.*

* E. A. Cassell, F. T. Sulzer, and J. C. Lamb, III, "Population Dynamics and Selection in Continuous Mixed Culture," Sewage and Industiral Wastes, 38, No. 9, 1398-1409 (1966), p. 1398. 
Thus, although Monod's theory proved applicable to systems involving pure cultures, it does not seem adequate for mixed microorganism systems. The purpose of the present work is to modify Monod's theory to include the effects of predators. This results in an equation for the limiting nutrient concentration, an equation for the bacterium concentration, as well as an equation for a predator population. The solutions of the differential equations will be studied for dynamic phenomena.

\section{CLASSICAL PREDATOR-PREY RELATIONSHIPS}

Volterra ${ }^{17}$ and Lotka ${ }^{18}$ were the first to introduce mathematical models to aid in the understanding of predator-prey relationships. Consider a population of host organisms $N_{1}$ that has an effective growth rate of $k_{1}$. For simplicity, $k_{1}$ is assumed to be constant. This implies that the food supply of the host organisms is in excess. Next, it is hypothesized that the predator organism $N_{2}$ decreases the number of host organisms. Again, for simplicity, it is assumed that the decrease in the number of $N_{1}$ is proportional to $N_{1}$ and $N_{2}$. This leads to eq. (8), which expresses the change in the number of $N_{1}$ as a function of $N_{1}$ and $N_{2}$.

$$
\frac{d N_{1}}{d t}=k_{1} N_{1}-k_{2} N_{1} N_{2}
$$

The growth of the predator depends upon both the number of $N_{1}$ available and the number of $N_{2}$ present. If a simple proportional relationship is assumed with a proportionality constant of $k_{3}$, then eq. (9) expresses the change in the number of predators:

$$
\frac{d N_{2}}{d t}=k_{3} N_{1} N_{2}-k_{4} N_{2}
$$

where $-k_{4} N_{2}$ represents the death rate of the predators.

If eq. (8) is divided by eq. (9),

$$
\frac{d N_{1}}{d N_{2}}=\frac{\left(k_{1}-k_{2} N_{2}\right) N_{1}}{\left(k_{3} N_{1}-k_{4}\right) N_{2}}
$$

which integrates to eq. (11):

$$
-k_{4} \log N_{1}+k_{3} N_{1}-k_{1} \log N_{2}+k_{2} N_{2}=\text { constant }
$$


Each value of the integration constant gives a closed cycle for the relationship between $N_{1}$ and $N_{2}$ on the $N_{1}-N_{2}$ plane. The equilibrium value of $N_{1}$ is given by $k_{4} / k_{3}$. Similarly, the equilibrium value of $N_{2}$ is given by $k_{1} / k_{2}$. A plot of $N_{1}$ versus $N_{2}$ for different values of the integration constant gives closed cycles as shown in Figure 1.

Biologists as well as mathematicians have shown considerable interest in Lotka's theory since it was proposed in 1924. Gause ${ }^{19}$ was the first to show the actual existence of cyclic variation between host and predator organisms. Leslie ${ }^{20}$ and Bartlett ${ }^{21}$ discussed the validity of the theory and introduced the use of stochastic population models for predator-prey relationships. Salt ${ }^{22}$ discussed in great detail predation in a protozoan population. Cyclic population data were obtained for a case where Paramecium aurelia served as a host organism for Woodruffia metabolica. However, experimental observation of cyclic variations among predators and their prey has been rare. To this writer's knowledge, no attempts have been made to observe the phenomenon in a system where free-swimming holozoic protozoa prey on bacteria. The demonstration of cyclic variations between such organisms will be of considerable interest to those concerned with biological waste treatment problems.

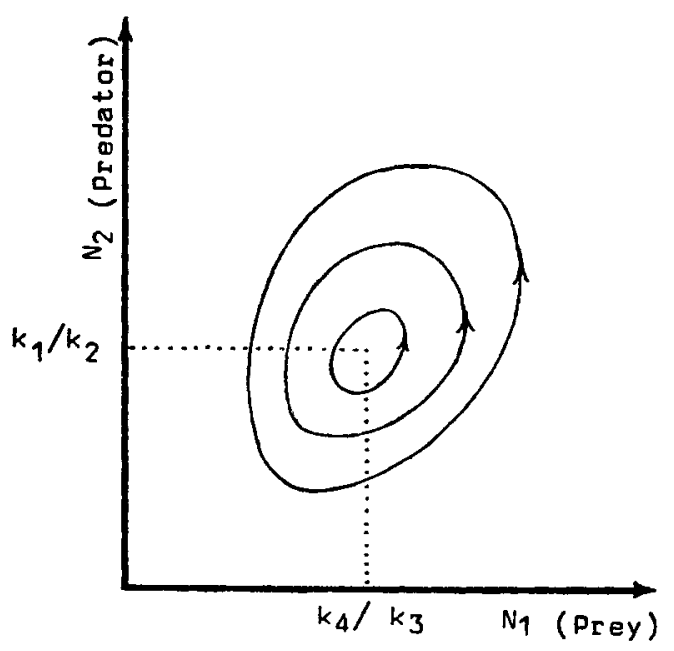

Fig. 1. Closed cycle relationship between predator and prey from classical theory. BIOTECHNOLOGY AND BIOENGINEERING, VOL. XI, ISSUE 5 
Equation (9) shows that the growth of the predator is proportional to the number of host and predator organisms. When the host organisms are very high, a corresponding high growth rate is proposed for the predator without a saturation effect. This is probably the weakest point in the mathematical formulation of Lotka. Later analysis points out how this shortcoming can easily be overcome.

\section{MATHEMATICAL DEVELOPMENT OF PREDATOR-PREY RELATIONSHIPS IN A CONTINUOUS FLOW STIRRED TANK BIOLOGICAL REACTOR}

\section{Derivation of Equations}

Equations to describe the variations in the mass of bacterium and the number of protozoan, and consequently, the concentration of the limiting nutrient, can be developed using some of the principles presented earlier. However, some of the simplifying assumptions made previously have been eliminated in the present work. These considerations demonstrate phenomena as yet unrevealed by other investigators.

Consider a stirred tank reactor with volume $V$. A sterile growth medium, which contains a limiting nutrient at concentration $s_{I}$, is continuously pumped into the tank with a volumetric flow rate $Q$. The reactor contains a pure culture of bacterium utilizing the nutrient medium as a source of food, and a pure culture of holozoic, freeswimming ciliated protozoan. The concentration of bacterium is represented by $b$ and the protozoan concentration by $p$. A mass balance on the limiting nutrient, assuming that environmental factors such as temperature, $\mathrm{pH}$, and toxicity are constant, is expressed as eq. (12), which is identical to eq. (4):

Rate of change of

Rate of

limiting nutrient $=$ Rate of - Rate of - consumption by concentration input output bacteria

$$
\frac{d s}{d t} \quad=\frac{Q}{V} s_{I}-\frac{Q}{V} s-\frac{M}{Y} \frac{s}{K+s} b
$$

A material balance on the mass concentration of the bacterium, 
neglecting death and endogenous respiration rates, gives eq. (13):

Rate of change of Rate of concentration of $=$ Rate of - Rate of - consumption by bacterium growth output protozoan

$$
\frac{d b}{d t} \quad=M \frac{s}{K+s} b-\frac{Q}{V} b-f(b, p)
$$

The rate of bacterium consumption by the protozoan is given by some unknown function, $f(b, p)$. A material balance on the number concentration of protozoan, neglecting death rate, gives eq. (14):

Rate of change

of protozoan = Rate of growth - Rate of output concentration

$$
\frac{d p}{d t} \quad=\quad g(b, p) \quad-\quad \frac{Q}{V} p
$$

The rate of protozoan growth is described by an unknown function, $g(b, p)$.

It is seen that further progress is impossible without some experimental work to describe the functions $f(b, p)$ and $g(b, p)$. However, Proper and Garver, ${ }^{23}$ working with a monoxenic culture of Colpoda steinii and Escherichia coli, have shown that protozoan growth can be described by Michaelis-Menten kinetics considering the bacterium concentration as the limiting nutrient. Further, these workers have shown that the protozoan yield $W$ is proportional to the amount of bacterium initially present, where:

$$
W=\frac{\text { number of protozoan produced }}{\text { mass of bacterium consumed }}
$$

Thus, eqs. (16) and (17) can be used to describe $f(b, p)$ and $g(b, p)$ :

$$
\begin{aligned}
& f(b, p)=\frac{N}{W} \frac{b}{L+b} p \\
& g(b, p)=N \frac{b}{L+b} p
\end{aligned}
$$

where $N$ is the specific growth rate for the protozoan and has units BIOTECHNOLOGY AND BIOENGINEERING, VOL. XI, ISSUE 5 
of time $e^{-1}$, and $L$ is a saturation constant and is the concentration of bacterium when $g(b, p) / p=N / 2$. Combining eqs. (12), (13), and (14) and eqs. (16) and (17), and introducing the dimensionless variables:

$$
\begin{array}{ll}
x=s / s_{I} & y=b / s_{I} Y \\
z=p / s_{I} Y W & \theta=Q t / V
\end{array}
$$

gives eqs. (18):

$$
\begin{aligned}
& \frac{d x}{d \theta}=1-x-A \frac{x}{a+x} y \\
& \frac{d y}{d \theta}=A \frac{x}{a+x} y-y-B \frac{y}{d+y} z \\
& \frac{d z}{d \theta}=B \frac{y}{d+y} z-z
\end{aligned}
$$

where $a=K / s_{I}, d=L / s_{I} Y, A=M V / Q$, and $B=N V / Q$. Equations (18) are similar to models suggested by Bungay and Bungay ${ }^{24}$ and Drake et al..$^{25}$

\section{Solution of Equations}

In general, given an initial condition, the solution of eqs. (18) will trace a curve in the $x-y-z$ space. Figure 2 shows a typical variation of $x, y$, and $z$ obtained from an numerical solution of eqs. (18) with $A=7.5, B=2.4, a=.19$, and $d=.20$. However, it can be shown that for any given initial condition, the sum $(x+y+z)$ always approaches unity. Let $s^{\prime}=x+y+z$, and add eqs. (18) to obtain:

$$
d s^{\prime} / d \theta=1-s^{\prime}
$$

the solution of which is: $s^{\prime}=1-\left(1-K^{\prime}\right) \mathrm{e}^{-\theta}$, where $K^{\prime}$ is the initial value of the sum $s^{\prime}$. All the solution trajectories in three-dimensional space approach the plane $x+y+z=1$ at $\theta$ approaches infinity. Further, if $s^{\prime}=x+y+z=1$ to start with, then $d s^{\prime} / d \theta$ equals zero, and the solution trajectories remain on the plane $x+y+z=1$. Thus, the characteristics of the solutions of the three-variable problem represented by eq. (18) can be studied as a two-variable 


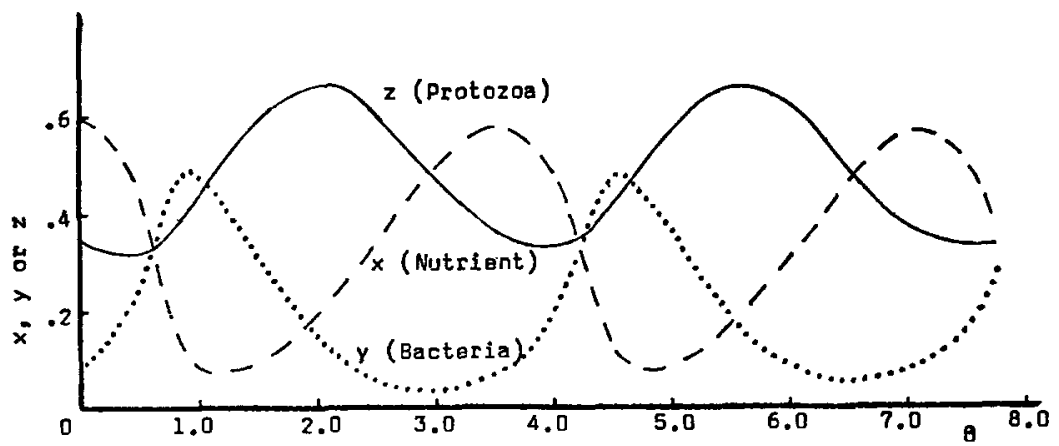

Fig. 2. Variation of predator, prey, and nutrients with $\theta$ according to eqs. (18).

problem on the plane $x+y+z=1$. Assuming the initial condition $x+y+z=1$, eqs. (18) can be reduced to eqs. (20):

$$
\begin{aligned}
& \frac{d y}{d \theta}=\frac{A(1-y-z) y}{a+1-y-z}-y-\frac{B y z}{d+y} \\
& \frac{d z}{d \theta}=\frac{B y z}{d+y}-z
\end{aligned}
$$

The singular points of eqs. (20) are obtained by setting $(d y / d \theta)=$ $(d z / d \theta)=0$. There are three singular points in the system.

$$
\begin{aligned}
& \text { Singular }\left\{\begin{array} { l } 
{ y _ { 0 } = \frac { d } { B - 1 } } \\
{ \text { Point AA } }
\end{array} \left\{\begin{array}{l}
x_{0}=-\frac{1}{2}\left(A y_{0}+a-1\right)+\frac{1}{2} \sqrt{\left(A y_{0}+a-1\right)^{2}+4 a} \\
z_{0}=1-x_{0}-y_{0}
\end{array}\right.\right. \\
& \text { Singular }\left\{\begin{array}{l}
y_{0}=0 \\
z_{0}=0 \\
x_{0}=1
\end{array}\right.
\end{aligned}
$$$$
\text { Singular }\left\{\begin{array}{l}
z_{0}=0 \\
y_{0}=\frac{A-a-1}{A-1} \\
x_{0}=\frac{a}{A-1}
\end{array}\right.
$$

To study the nature of each of these singular points, use it as an BIOTECHNOLOGY AND BIOENGINEERING, VOL. XI, ISSUE 5 
origin and define a new pair of variables, $u=y-y_{0}$ and $v=z-z_{0}$. With $u$ and $v$ as dependent variables, eqs. (20) become:

$$
\begin{aligned}
& \dot{u}=\frac{A\left(1-u-v-y_{0}-z_{0}\right)\left(u+y_{0}\right)}{a+1-u-v-y_{0}-z_{0}}-u-y_{0} \\
& \dot{v}=\frac{B\left(u+y_{0}\right)\left(v+z_{0}\right)}{d+u+y_{0}} \\
& d+u+y_{0}-v-z_{0}
\end{aligned}
$$

In the region of $y_{0}$ and $z_{0}, u$ and $v$ are always small and the nature of the solutions of eqs. (21) can be investigated with linear approximating equations near the singular points making use of well known theorems from nonlinear analysis. Near the singular point AA, the linear approximation becomes (where $\dot{u}$ and $\dot{v}$ represent first derivatives with respect to $\theta$ ):

$\left[\begin{array}{c}\dot{u} \\ \\ \dot{v}\end{array}\right]$

$$
=\left[\begin{array}{cc}
\frac{1-x_{0}-A y_{0}+A x_{0}}{a+x_{0}}-1+\frac{z_{0}(1-B)}{d+y_{0}} & \frac{1-x_{0}-A y_{0}}{a+x_{0}}-1 \\
\frac{-z_{0}(1-B)}{d+y_{0}} & 0
\end{array}\right]\left[\begin{array}{l}
u \\
v
\end{array}\right]
$$

Near the singular point BB, the linear equation becomes:

$$
\left[\begin{array}{c}
\dot{u} \\
\dot{v}
\end{array}\right]=\left[\begin{array}{cc}
\frac{A-z-1}{a+1} & 0 \\
0 & -1
\end{array}\right]\left[\begin{array}{l}
u \\
v
\end{array}\right]
$$

Near the singular point $\mathrm{CC}$, the linear equation becomes:

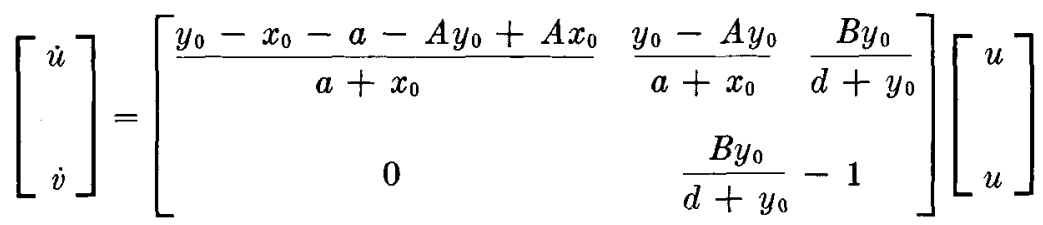


The elements of the coefficient matrices in eqs. (22), (23), and (24) depend on the four parameters; $A, B, a$, and $d$. Therefore, the numberical values of $A, B, a$, and $d$ determine the nature of the solution of eqs. (20). By selecting $A=30, a=.02$, and $d=.1$ and varying the numerical value of $B$, it is possible to demonstrate three types of solutions.

If $A=30, a=.02, d=.1$, and $B=2$, both eigenvalues of eq. (22) are real and negative. The eigenvalues of eqs. (23) and (24) are real but are of opposite sign. Therefore, the singular point at AA is a stable node, while the singular points at $\mathrm{BB}$ and $\mathrm{CC}$ are saddle points. Figure 3c (not to scale) shows a sketch of the solution trajectories for this case on the $x+y+z=1$ plane.

On the $x-y$ plane, where $z=0$ for all $\theta$, eqs. (18) reduce to eqs. (25).

$$
\begin{aligned}
& \dot{x}=1-x-A \frac{x}{a+x} y \\
& \dot{y}=A \frac{x}{a+x} y-y
\end{aligned}
$$

The singular points of eqs. (25) are obtained by setting $\dot{x}=\dot{y}=0$. This system has two singular points.

$$
\begin{aligned}
& \text { Singular } \begin{array}{l}
y_{0}=0 \\
\text { Point aa }
\end{array}\left\{\begin{array}{l}
x_{0}=1
\end{array}\right. \\
& \begin{array}{l}
\text { Singular } \\
\text { Point } \mathrm{bb}
\end{array} \quad\left\{\begin{array}{l}
y_{0}=1-x_{0} \\
x_{0}=a /(A-1)
\end{array}\right.
\end{aligned}
$$

Again introduce the small variables $u$ and $v$, and expand eqs. (25). The eigenvalues of the resulting linear approximations show that
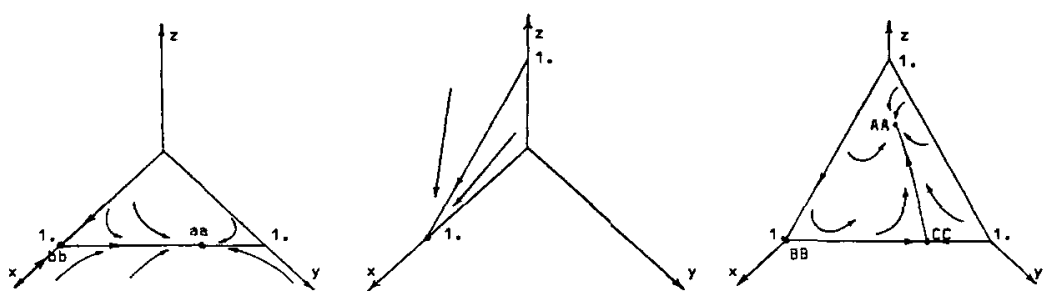

Fig. 3. Solution trajectories for stable node at singular point AA. BIOTECHNOLOGY AND BIOENGINEERING, VOL. XI, ISSUE 5 
singular point aa is a saddle point and that singular point $\mathrm{bb}$ is a stable node provided that $A /(a+1)$ is greater than 1 . Figure 3a (not to scale) shows a sketch of the solution trajectories on the $x-y$ plane.

On the $x$ - $z$ plane, where $y=0$ for all $\theta$, eqs. (18) reduce to eqs. (26).

$$
\begin{aligned}
& \dot{x}=1-x \\
& \dot{z}=-z
\end{aligned}
$$

The solutions of eqs. (26) are a series of straight lines:

$$
z=\frac{\bar{z}}{\bar{x}-1} x+\frac{\bar{z}}{1-\bar{x}}
$$

where $z(0)=\bar{z}$ and $x(0)=\bar{x}$. Figure 3b shows a sketch of these lines on the $x-z$ plane.

The singular point AA is a stable node if $A=30, a=.02, d=.1$, and $B=2$. By setting $A=30, a=.02, d=.1$, and $B=3$, a different type of solution is obtained. The eigenvalues of eqs. (22) become complex conjugates with negative real parts, while the eigenvalues of eqs. (23) and (24) are real but of opposite sign. Thus, singular point AA becomes a stable focal point. The singular points $\mathrm{BB}$ and $\mathrm{CC}$ remain saddle points. Figure 4 (not to scale) shows a sketch of the solution trajectories for this situation on the $x+y+z=1$ plane.

Examination of eqs. (22), (23), and (24) when $A=30, a=.02$, $d=.1$, and $B=4$, shows that the eigenvalues of eq. (22) are complex conjugates with positive real parts, while the eigenvalues of eqs. (23) and (24) are real but of opposite sign. Singular point AA becomes an unstable focal point. Singular points $\mathrm{BB}$ and $\mathrm{CC}$ are saddle points. Figure 5 (not to scale) shows a sketch of the solution trajectories when singular point AA si an unstable focal point.

When singular point AA is an unstable focal point, the solution trajectories of eq. (18) eventually approach a closed curve on the $x+y+z=1$ plane. This closed curve is a periodic solution of eq. (18) and is called a limit cycle. The shape and frequency of the waves of this solution are independent of the initial conditions and depend only on the values of $A, B, a$, and $d$.

Qualitative information of the effects of bacterium and protozoan growth rates, as well as the effects of the saturation constants, can be 


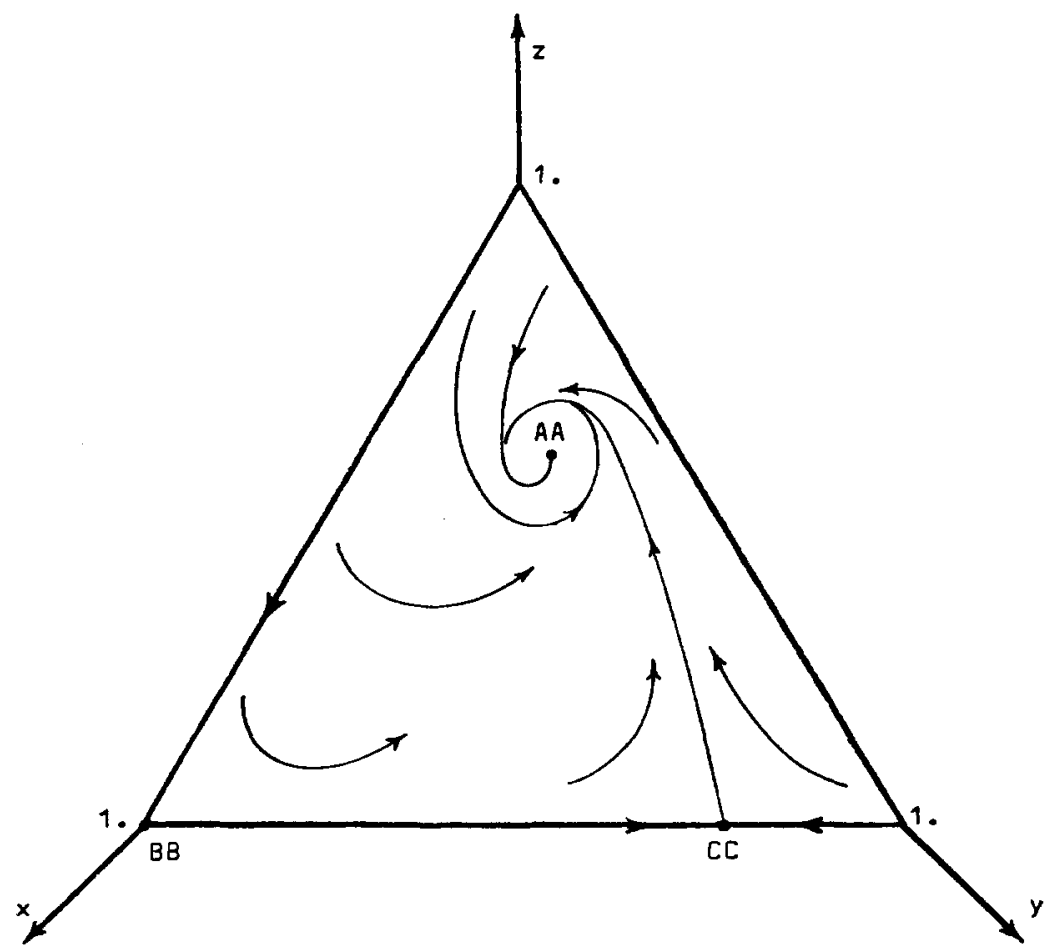

Fig. 4. Solution trajectories for stable focal point at AA.

obtained by examining eqs. (20). Figures 6 through 9 are phaseplane sketches of $y$ versus $z$ obtained from numerical solutions of eqs. (20) by varying each of the constants $A, B, a$, and $d$, while fixing the other three.

Figure 6 shows that increasing the bacterial growth rate increases the average population of protozoan, while decreasing the concentration of the limiting nutrient. Systems with very high bacterial growth rates may be subject to high frequency, low amplitude oscillations.

Figure 7 shows that increasing the saturation constant determining bacterial growth increases the period of the oscillations. Lower average populations of protozoan are possible, while limiting nutrient concentrations are higher. 
Figure 8 shows the effect of varying the protozoan maximum growth rates. For low maximum protozoan growth rates, it is possible for the oscillations to degenerate into either stable focal or stable nodal points. At these points, the average protozoan population will be extremely high, while the bacterium and limiting nutrient concentrations will be low. Increasing the maximum growth rate pushes the system into a low frequency, high amplitude oscillation.

Figure 9 shows the effect of varying the saturation constant for protozoan growth. For cases with high saturation constants, the system may reduce to a stable nodal point with a high equilibrium concentration of protozoan, and correspondingly low equilibrium concentrations for the bacterium and limiting nutrient. Decreasing the saturation constant leads to oscillations with extremely high amplitude and very low frequencies.

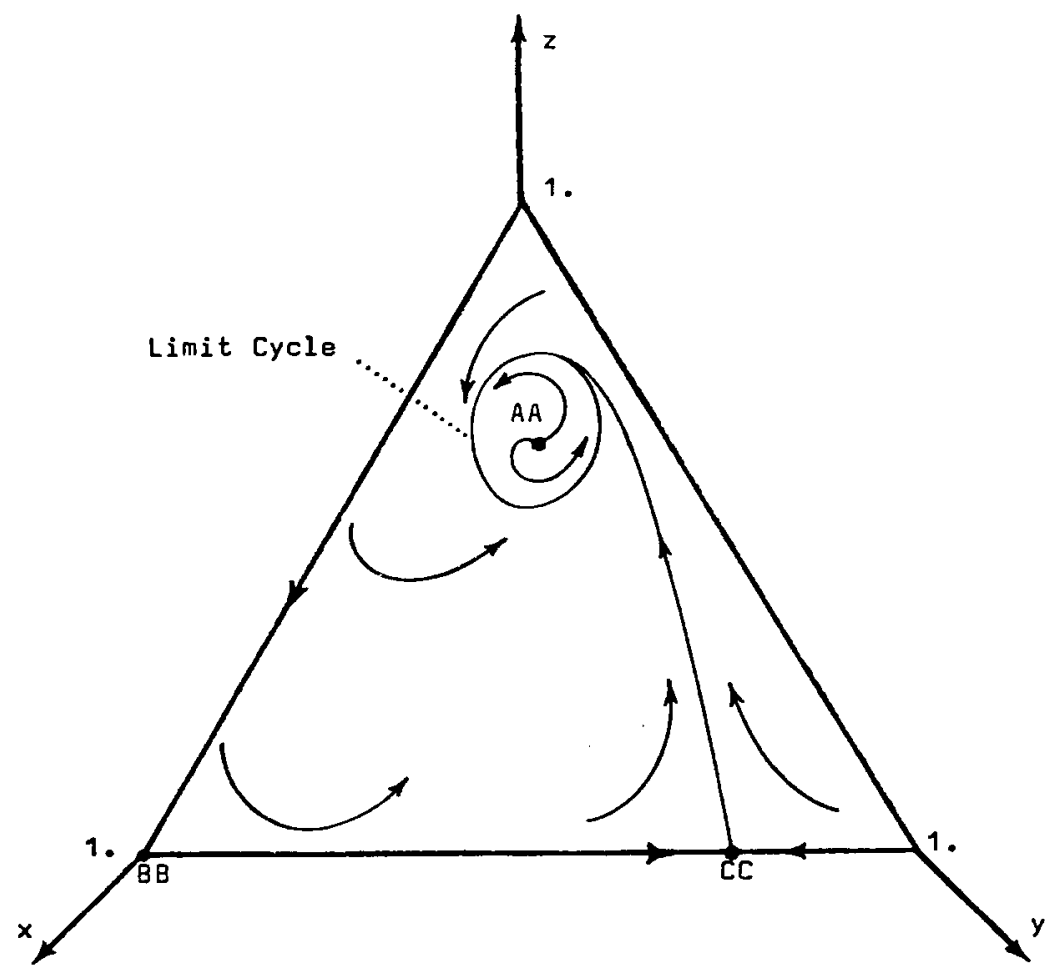

Fig. 5. Solution trajectories for unstable focal point AA. 


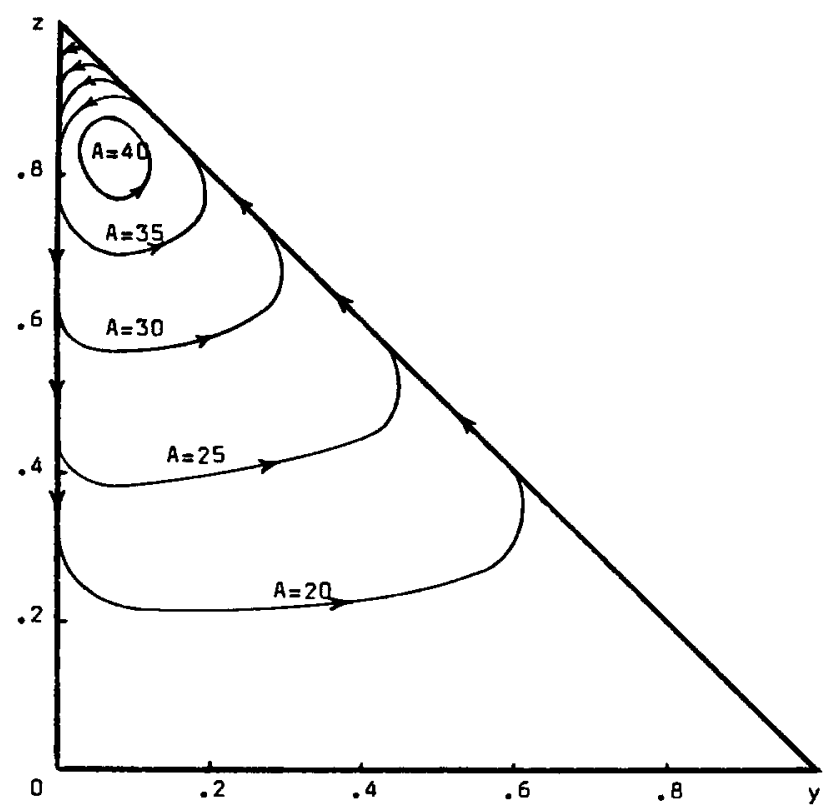

Fig. 6. Theoretical effect of varying bacterium maximum growth rate according to eqs. (18). $\mathrm{y}-\mathrm{z}$ plane. $\mathrm{B}=4.0, \mathrm{a}=.02$, and $\mathrm{b}=.1$.

\section{SUMMARY AND CONCLUSIONS}

The primary objective of this research is to obtain a quantitative approach to aid in understanding the food chain,

$$
\begin{gathered}
\text { Soluble Organic } \\
\text { Nutrients }
\end{gathered} \text { Bacteria } \longrightarrow \begin{gathered}
\text { Holozoic } \\
\text { Protozoa }
\end{gathered}
$$

The interdependence of the elements in this food chain give rise to dynamic phenomena. Lotka and Volterra were the first investigators to use mathematics as a tool to analyze these dynamic phenomena. These workers introduced eqs. (8) and (9) as an explanation of observed periodic oscillations in numbers of host and predator organisms:

$$
\begin{aligned}
& \frac{d N_{1}}{d t}=k_{1} N_{1}-k_{2} N_{1} N_{2} \\
& \frac{d N_{2}}{d t}=k_{3} N_{1} N_{2}-k_{4} N_{2}
\end{aligned}
$$

BIOTECHNOLOGY AND BIOENGINEERING, VOL. XI, ISSUE 5 
The solutions of these differential equations can be represented as a set of closed curves on the $N_{1}, N_{2}$ plane. The amplitude and frequency of the oscillations depends on the initial conditions. However, Gause (1935) discussed the fact that when periodic oscillations arise, the amplitude and frequency are independent of the initial conditions and seem to depend on the nature of the system. Equation 8 assumes that the source of food for the host organisms is unlimited. Equation 9 assumes that the growth of the predator is strictly proportional to the number of hosts without any saturation effect.

The present investigation led to a set of three differential equations to describe the interrelationships among soluble organic nutrients, saprophytic prey (bacterium), and holozoic predators (protozoan) in a continuously fed, stirred tank biological reactor. The differential equations do not neglect the food source of the prey. Saturation of the growth rate of the predator is also considered. The final form of

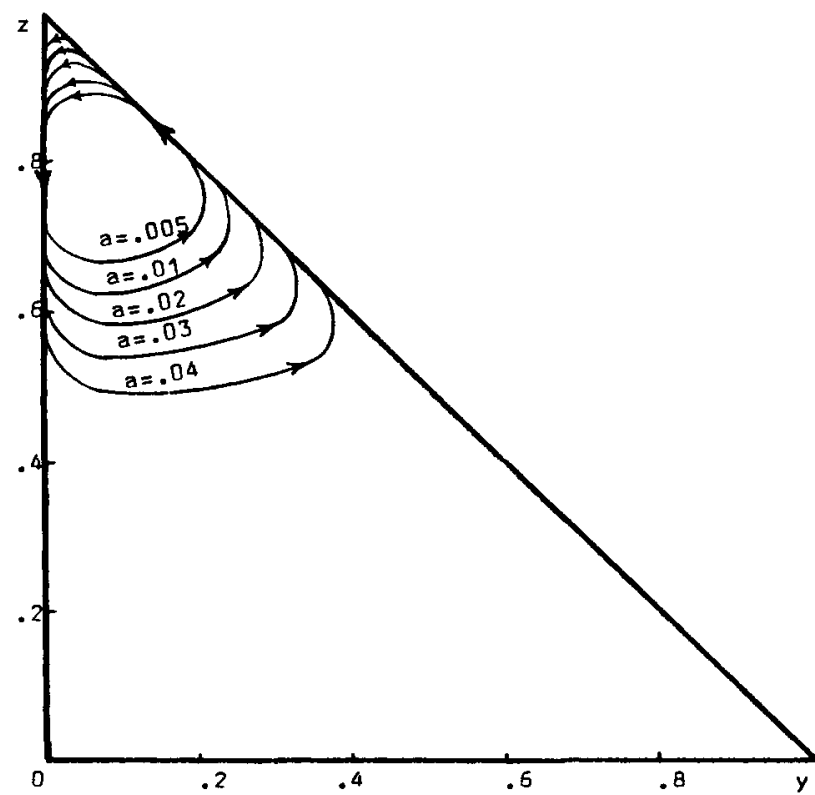

Fig. 7. Theoretical effect of varying saturation constant determining bacterium growth rate according to eqs. (18). y-z plane. $\mathrm{A}=30, \mathrm{~B}=4$, and $\mathrm{b}=0.1$. 


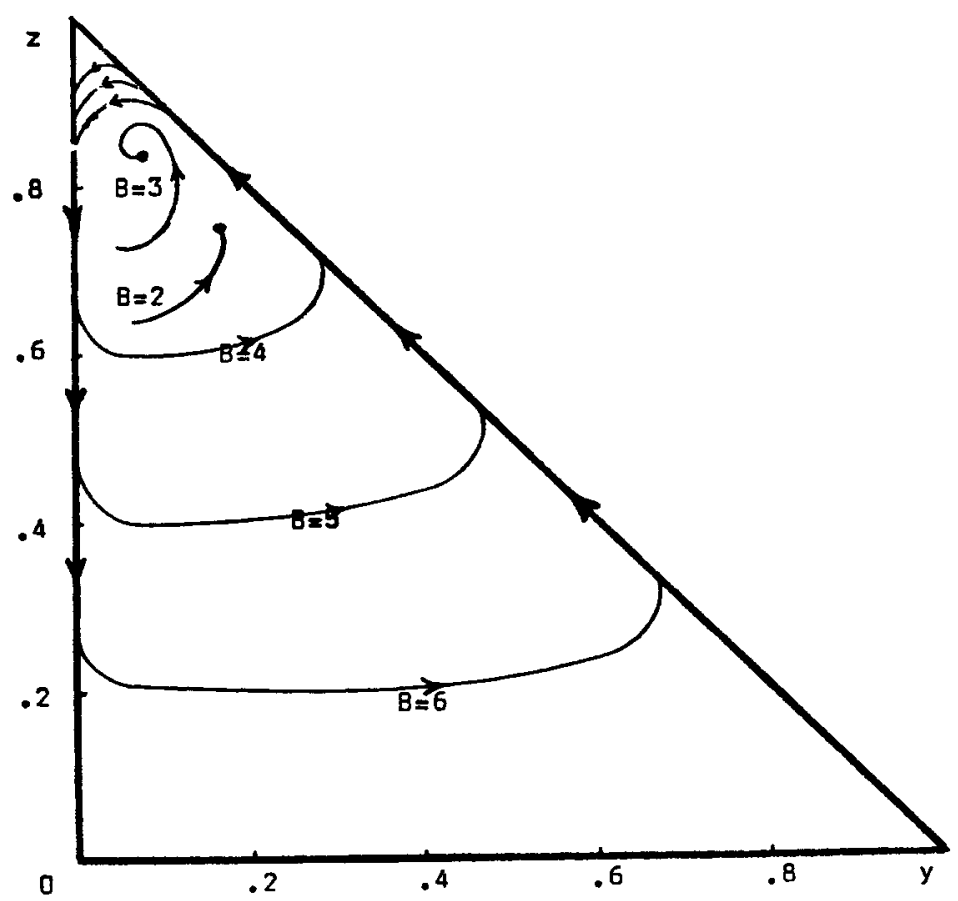

Fig. 8. Theoretical effect of varying protozoan maximum growth rate according to eqs. (18). y-z plane. $A=30, a=0.02$, and $b=0.1$.

the proposed differential equations is given by eqs. (18):

$$
\begin{aligned}
& \frac{d x}{d \theta}=1-x-A \frac{x}{a+x} y \\
& \frac{d y}{d \theta}=A \frac{x}{a+x} y-y-B \frac{y}{d+y} z \\
& \frac{d z}{d \theta}=B \frac{y}{d+y} z-z
\end{aligned}
$$

This model has also incorporated several simplifying assumptions and therefore should be regarded as one of several plausible alternatives. The need for experimental evidence is apparent.

Equations (18) are highly nonlinear. The characteristics of any solution of such equations depend on the magnitude of the parameters BIOTECHNOLOGY AND BIOENGINEERING, VOL. XI. ISSUE 5 


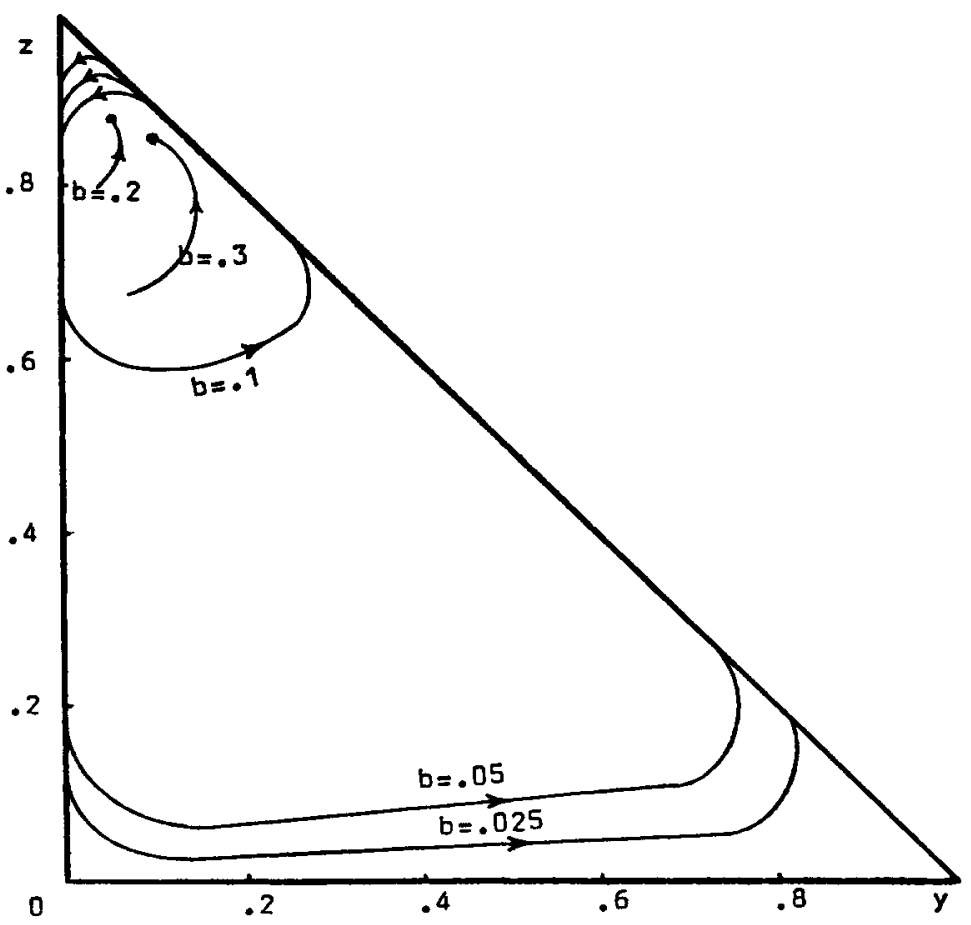

Fig. 9. Theoretical effect of varying saturation constant determining protozoan growth rate according to eqs. (18). $y-z$ plane. $A=30, a=0.02$, and $B=4$.

in the system. As shown by singular point analysis and numerical integration, the solution of these equations may be of three possible types: (1) a stable limit cycle variation among $x, y$, and $z$ where the amplitude and period depend of the parameters $A, B, a$, and $d$ and not on the initial conditions; (2) a damped oscillation variation among $x, y$, and $z$ which eventually decays to some nonzero values; (3) a nonoscillatory variation among $x, y$, and $z$ which asymptotically approaches constant values.

An experimental program designed to test the validity of eqs. (18) is being conducted. Tetrahymena pyriformis $W$ is serving as a predator on Aerobacter aerogenes in a buffered cerophyl-glucose solution. A report of these findings will be presented at a later date. Experimental verification of the proposed predator-prey models 
developed in this paper will be of extreme value to those interested in the dynamics of the activated sludge system.

The author is indebted to the staff of the Department of Civil Engineering at Syracuse University for their support of this research. Particular thanks are extended to Dr. Paul A. Brennan (Department Chairman), Dr. Wen-Hsiung Li, and Dr. M. C. Rand for their individual contributions.

\section{References}

1. J. B. Lackey, "Biology of Sewage Treatment," Sewage Works Journal, 21, (4), 649 (1949).

2. S. H. Jenkins, "Role of Protozoa in the Activated Sludge Process," Nature, 150, 607 (1942).

3. S. Baines, H. A. Hawkes, C. H. Hewitt, and S. H. Jenkins, "Protozoa as Indicators in Activated Sludge Treatment," Sewage and Industrial Wastes, 25, No. 9, 1023-1033 (1953).

4. R. E. McKinney and A. Gram, "Protozoa and Activated Sludge," Sewage and Industrial Wastes, 28, 1219-1231 (1956).

5. R. Cramer, "The Role of Protozoa in Activated Sludge," Ind. Eng. Chem., 23, 309-313 (1931).

6. T. B. Reynoldson, "Vorticella as an Indicator Organism for Activated Sludge," Nature, 149, 3787, 608-609 (1942).

7. H. Haukelekian and M. Gurbaxani, "Effect of Certain Physical and Chemical Agents on the Bacteria and Protozoa of Activated Sludge," Sewage Works Journal, 21, No. 5, 811-817 (1949).

8. D. Herbert, R. Elsworth, and R. C. Telling, "The Continuous Culture of Bacteria; a Theoretical and Experimental Study," J. Gen. Microbiol., 14, 601-622 (1956).

9. A. L. Downing and A. B. Wheatland, "Fundamental Considerations in Biological Treatment of Effluent," Industrial Engineering Chemistry, 4, No. 2, 91 (1962).

10. M. J. Stewart and H. F. Ludwig, "Theory of the MAS Waste Water Treatment Process, Parts I and II," Water and Sewage Works, 109, Nos. 2 and 3 (1962).

11. L. C. Rich, Unit Processes of Sanitary Engineering, John Wiley and Sons, Inc., New York, 1963.

12. S. Koga and A. E. Humphrey, "Study of the Dynamic Behavior of the Chemostat System," Biotechnology and Bioengineering, 9, 375-386 (1967).

13. E. J. Martin and D. R. Washington, "Kinetics of Steady-State Bacterial Culture. I-Mathematical Model," Proceedings of the 19th Purdue Industrial Waste Conference, May, 1963, pp. 724-737.

14. D. R. Washington, "Kinetics of the Steady State Bacterial Culture with Applications to the Activated Sludge Process," Final Report of the Environmental Engineering Department at Rensselear Polytechnic Institute, Troy, New York, 1965.

15. T. D. Reynolds, and J. T. Yang, "Model of the Completely-Mixed Activated Sludge Process," Proceedings of the 21st Purdue Industrial Waste Conference, Part II, 1966, pp. 696-713. 
16. E. A. Cassell, F. T. Sulzer, and J. C. Lamb III, "Population Dynamics and Selection in Continuous Mixed Cultures," Sewage and Industrial Wastes, 38, No. 9, 1398-1409 (1966).

17. V. Volterra, "Variazioni e fluttuazioni del numers d'individui in specie animali conviventi," Mem. Acad. Lineii Roma, 2, 31 (1926).

18. A. J. Lotka, Elements of Physical Biology, Williams and Wilkins, Baltimore, Maryland, 1924 (Reprinted: Dover, New York, 1956).

19. G. F. Gause, The Struggle for Existence, Williams and Wilkins, Baltimore, Maryland, 1934 (Reprinted: Hafner, New York, 1964).

20. P. H. Leslie and J. C. Gower, "The Properties of a Stochastic Model for Two Competing Species," Biometrika, 45, 316-330 (1958).

21. M. S. Bartlett, "On Theoretical Models for Competitive and Predatory Biological Systems," Biometrika, 44, 27-42 (1957).

22. G. W. Salt, "Predation in an Experimental Protozoan Population (Woodruffin-Paramecium)," Ecological Monographs, 37, 2, 113-114 (1967).

23. G. Proper and J. C. Garver, "Mass Culture of the Protozoa Colpoda steinii," Biolechnology and Bioengineering, VIII, 287-296 (1966).

24. H. R. Bungay, III and M. L. Bungay, "Microbial Interactions in Continuous Culture," Advances in Applied Microbiology, 10, 269-290 (1968).

25. J. F. Drake, J. L. Jost, A. G. Fredrickson, and H. M. Tsuchiya, "The Food Chain," Bioregenerative Systems, A conference held in Washington, D.C., November 15-16, 1966 sponsored by NASA and the American Institute of Biological Sciences, NASA SP-165, Library of Congress number 68-60345, U.S. Government Printing Office, Washington, D.C., 1966.

Received March 15, 1969

Revised May 23, 1969 